\title{
THE ROLE OF THE MULTI BUFFER LAYER TECHNIQUE ON THE STRUCTURAL QUALITY OF GaN
}

\author{
M. Benamara, Z. Liliental-Weber, J.H. Mazur ${ }^{+}$, W. Swider \\ and J. Washburn. \\ Materials Science Division, Lawrence Berkeley National \\ Laboratory, Berkeley CA 94720, 62/203 \\ + present address: Philips Semiconductors, 9651 \\ Westoverhills Blvd., San Antonio, TX 78251 \\ M. Iwaya, I. Akasaki and H. Amano. \\ Meijo University, Dept. of Electrical and Electronic \\ Engineering, 1-501 Shiogamaguchi, Tempaku ku, Nagoya 468- \\ 8502, Japan.
}

\begin{abstract}
Successive growth of thick GaN layers separated by either LT-GaN or LT-AIN interlayers have been investigated by transmission electron microscopy techniques. One of the objectives of this growth method was to improve the quality of $\mathrm{GaN}$ layers by reducing the dislocation density at the intermediate buffer layers that act as barriers to dislocation propagation. While the use of LT-AIN results in the multiplication of dislocations in the subsequent GaN layers, the LT-GaN reduces dislocation density. Based upon Burgers vector analysis, the efficiency of the buffer layers for the propagation of the different type of dislocations is presented. LT-AIN layer favor the generation of edge dislocations, leading to a highly defective GaN layer. On the other hand, the use of LT-GaN as intermediate buffer layers appears as a promising method to obtain high quality GaN layer.
\end{abstract}

\section{INTRODUCTION}

$\mathrm{GaN}$ and related alloys are of particular interest since their ability to cover a wide spectral range [1] that is not possible with any combination of any other semiconductor materials. Since no cheap crystalline substrate with a lattice parameter close enough to that of GaN can be available, growth techniques have been improved in order to limit the defect density in the GaN layer. The most commonly used technique consists in the deposition of an AlN buffer layer to form the junction between the substrate and the GaN layer and leads to 
the formation of more than $10^{9}$ dislocations $/ \mathrm{cm}^{2}$. Elaborated techniques have since emerged. The so-called "lateral epitaxial overgrowth" LEO technique appears today to give the best results. The lowest dislocation density is observed at the top of the $\mathrm{GaN}$ layers despite the presence of grain boundaries with mixed dislocations to compensate a misorientation [2]. Another technique is to first deposit a thick low-temperature (LT) GaN on the substrate. The first stage of the growth gives 3-D islands of $\mathrm{GaN}$ on the substrate. Afterwards, deposition of $\mathrm{GaN}$ at high-temperature (HT) makes the growth follow the same principles as those for LEO. These two latter techniques are based upon the faster growth along the $\langle\overline{2} 110\rangle$ directions. In this paper, we report on a Transmission Electron Microscopy study of GaN layers obtained by using the deposition of successive LT-GaN layers between HT-GaN layers.

\section{EXPERIMENTAL}

GaN layers were grown on (0001) sapphire substrates in an horizontal reactor as described in ref [3]. TMGa, TMAl and amonia were used as $\mathrm{Ga}, \mathrm{Al}$ and $\mathrm{N}$ source respectively, at around 140 Torr growth pressure.

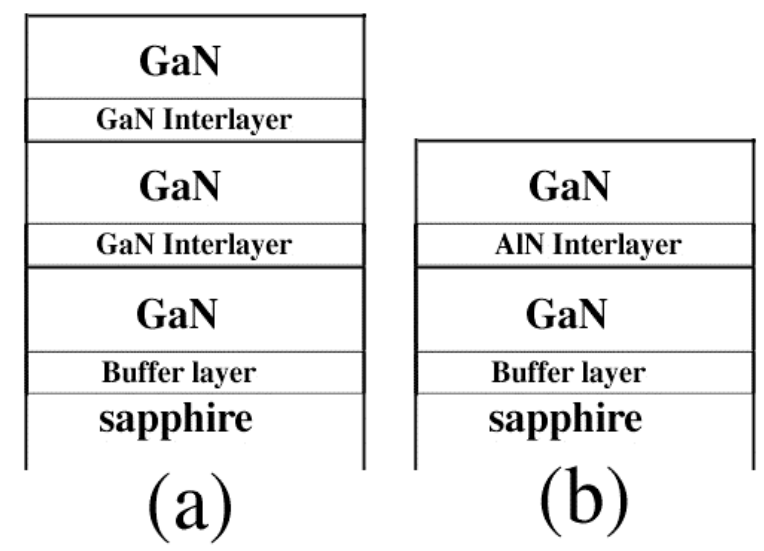

fig 1: Schematic drawing of the structure of the two studied samples

The structure of the samples used in this study is represented in fig. 1. A $1 \mu \mathrm{m}$ thick GaN layer is deposited at high-temperature (HT) over a low-temperature LT-GaN or LT-AlN buffer layer. Afterwards, a second buffer layer is deposited at low-temperature (LT). This second buffer layer is called interlayer and its goal is to improve the quality of the subsequent $1 \mu \mathrm{m}$ thick HT-GaN layer. It is a few nanometer thick AlN or GaN layer. For the investigations, the TEM samples were prepared by standard procedure using ion-milling at liquid 
nitrogen temperature and examined using a Jeol $200 \mathrm{CX}$ operating at $200 \mathrm{kV}$. Before the observations, the samples were mounted on a gold covered grid and then dipped in a $\mathrm{KOH}(50 \%)$ solution for $5 \mathrm{~min}$. This etch step was aimed at removing damage created during the ion milling.

\section{RESULTS}

\section{A- GaN/GaN interlayer/GaN}

Fig 2 is a dark field image of the sample the structure of which is represented in fig 1.a. The diffraction conditions were chosen so that all dislocations are visible in the image. It is noticed that the first HT-GaN layer is highly defective with a huge density of threading dislocations. Conventional diffraction contrast method was used to determine their Burgers vectors $\mathbf{b}$ using the invisibility criterion, $\mathbf{g} . \mathbf{b}=0$. Diffraction analysis showed that more than $80 \%$ of these dislocations have a Burgers vectors $\mathbf{b}=\mathbf{a}$. Their density is well above $10^{11} / \mathrm{cm}^{2}$.

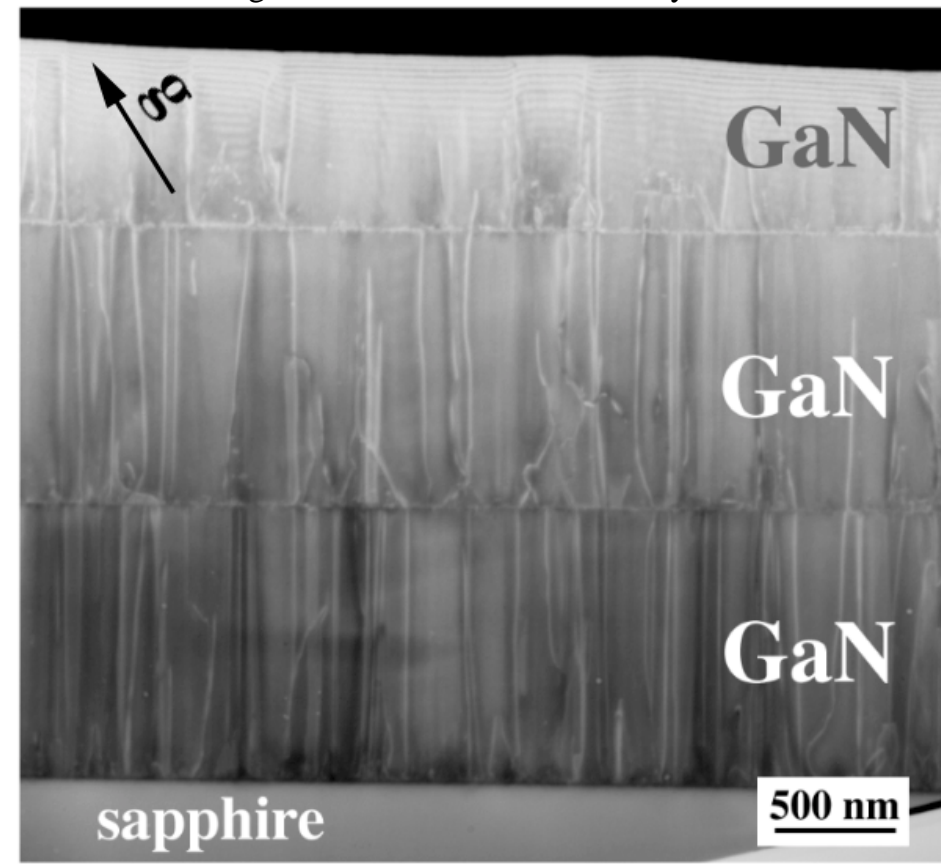

Fig 2: Dark field image of sample 1 with $\mathbf{g}=1 \overline{1} 02$. The density of dislocations decreases towards the surface.

The insertion of the first thin LT-GaN nucleation layer as a buffer layer does not allow one to obtain a high-quality HT-GaN layer. The large lattice 
mismatch with the sapphire substrate can be held responsible for such a high defect density.

This figure also shows a significant improvement has taken place in the subsequent GaN layer after deposition of the second intermediate LT-GaN layer. This second HT-GaN layer has a defect density of about $4.10^{9} \mathrm{~cm}^{-2}$. This confirms the role of the nucleation layer that acts as a barrier to dislocation propagation. Since dislocations in nitrides are frozen after the growth, the efficiency of the LT-GaN layer is mainly related to the growth process and the dislocation annihilation has taken place during the first growth stages of this LT-layer.

The density of dislocations in the third HT-GaN layer is around $1.4 \times 10^{9} \mathrm{~cm}^{-}$

2 . This density is three times smaller than in the second layer and it shows that the deposition of another LT-GaN layer on top of the second HT-GaN still benefits the technique. Nevertheless, this second intermediate LT-GaN layer is less efficient and this can be explained by the increasing quality of the GaN layer.

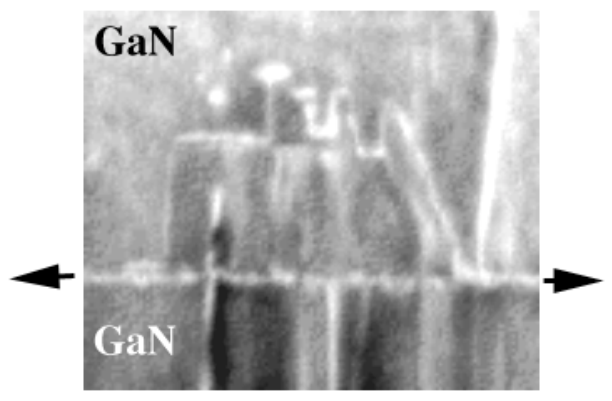

Fig.3: Dislocation half loop formed in the HT-GaN layer.

Dislocation half loops were observed with a high density in the vicinity of all LT-layer. They might be the result of lateral overgrowth of adjacent GaN islands, during which threading dislocations are forced to bend over into basal planes. Then their interaction with other dislocations often results in the formation dislocation half loops [4].

\section{B- GaN/AIN interlayer/GaN}

Fig 4 represents two weak-beam dark field images taken under different conditions. Fig 4.a. is taken with $1 \overline{1} 00$ reflecting planes and fig. $4 . b$ with $\mathrm{g}=0002$. Since the diffraction vector used on fig. $4 . \mathrm{a}$ is $\mathbf{g}=1 \overline{1} 00$, most of edge 
and mixed dislocations with Burgers vectors $\mathbf{b}=\mathbf{a}$ and $\mathbf{b}=\mathbf{a}+\mathbf{c}$ appear in the image (edge dislocations with $\mathbf{b}=1 / 3[11 \overline{2} 0]$ are out of contrast because $g . b=0$ and g.b $\wedge \mathrm{u}=0$ simultaneously).

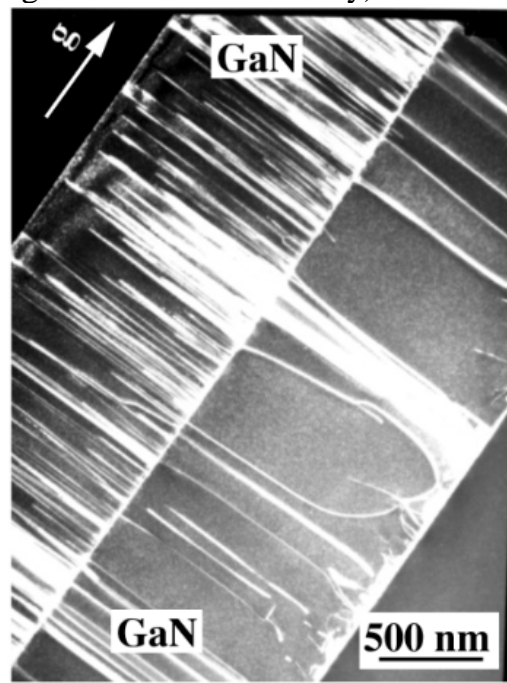

$\mathrm{g}=\mathbf{1 1 0 0 0}$

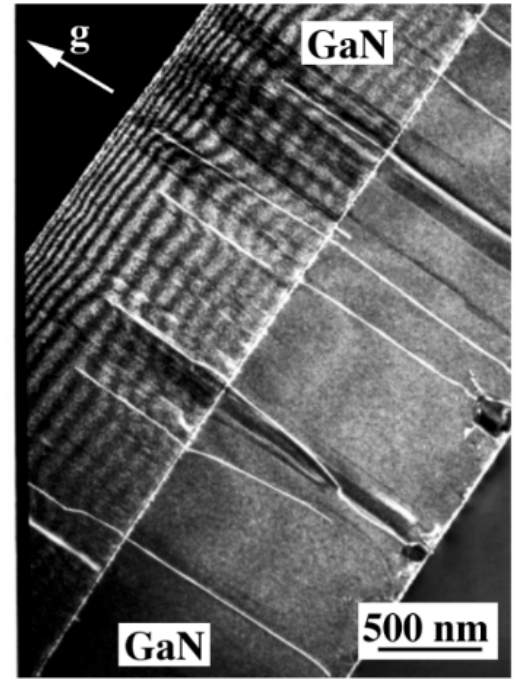

$\mathrm{g}=0002$

Fig. 4: Dark field images of the same area taken under different conditions (a) with $\mathbf{g}=1 \overline{1}$ 00: note the high density of edge dislocations in the upper layer. (b) with $\mathbf{g}=0002$, the upper layer appears defect free.

It is obvious that the use of the LT-AlN buffer layer that makes the junction with the sapphire substrate greatly improves the quality of the first GaN layer. The density of threading dislocations in this layer is about $2.10^{9} \mathrm{~cm}^{-2}$. Even though the quality of the first HT-layer depends on the growth conditions and especially its first stages, the first HT-GaN layer of this sample has a better crystalline quality than the first HT-GaN layer of sample 1 (where a LT-GaN buffer layer was used). This density of $2.10^{9} \mathrm{~cm}^{-2}$ is typical for MOCVD grown GaN layers on sapphire using LT-AlN buffer layer. Nevertheless, the AlN buffer layer inserted between the two HT-GaN layers does not reduce the dislocation density. While the density of screw and mixed dislocations $\left(10^{8}\right.$ $\mathrm{cm}^{-2}$ ) remain the same in both GaN layers, the second AlN buffer introduces edge dislocations with a high density in the upper layer (Fig. 4.a). 


\section{SUMMARY}

Successive growth of thick GaN layers separated by either LT-GaN or LTAlN layer have been investigated by transmission electron microscopy techniques. One of the objectives of this growth method was to improve the quality of GaN layers by reducing the dislocation density at the intermediate buffer layers that act as barriers to dislocation propagation. While the use of LT-AlN results in the multiplication of dislocations in the subsequent GaN layers, the LT-GaN reduces dislocation density. Based upon Burgers vector analysis, the efficiency of the buffer layers for the propagation of the different type of dislocations is presented and discussed. LT-AlN buffer layers favors the generation of edge dislocations, leading to a highly defective GaN layer. On the other hand, the use of LT-GaN as intermediate buffer layers appears as a promising method to obtain high quality GaN layer.

\section{ACKNOWLEDGMENT}

Work at E.O. Lawrence Berkeley Laboratory was supported by the Director, Office of Basic Science, Materials Science Division, U.S. Department of Energy, under the Contract No. DE-AC03-76SF00098. The use of the facility at the National Center for Electron Microscopy at E.O. Lawrence Berkeley National Laboratory is greatly appreciated. Work at Meijo University was partly supported by the Ministry of Education, Science, Sports and Culture of Japan (contract Nos 09450133, 09875083 and High-Tech Research Center Project), Japan Society for the Promotion of Science (JSPS) Research for the Future Program in the area of Atomic Scale Surface and Interface Dynamics under the project of "Dynamical Process and Control of Buffer Layer at the Interface of Highly-Mismatched System”.

\section{REFERENCES}

[1] S. Nakamura and G. Fasol, The blue Laser Diode, (Springer, Berlin, 1997).

[2] Z. Liliental-Weber, M. Benamara, W. Swider, J. Washburn, J. Park, P.A. Grudowski, C.J. Eiting and R. D. Dupuis, MRS Internet J. Nitride Semicond. Res. 4S1, G4.6 (1999)

[3] H. Amano, M. Iwaya, N. Hayashi, T. Kashima, M.Katsuragawa,T. Takeuchi, C.Wetzel and I.Akasaki, MRS Internet J. Nitride Semicond. Res. 4S1, G10.1 (1999).

[4] S. Ruvimov, Z. Liliental-Weber, J. Washburn, Y. Kim, G. S. Sudhir, J. Krueger and E. R. Weber, Mat. Res. Soc. Proc. Vol. 572, 295 (1999). 